\title{
Commentary on Child-Adult Differences in Muscle Activation-A Review
}

\author{
Thomas D. O'Brien \\ Bangor University
}

\author{
Neil D. Reeves, Vasilios Baltzopoulos, David A. Jones, \\ and Constantinos N. Maganaris \\ Manchester Metropolitan University
}

Comparing muscle strength and function between subjects differing in size, age, sex and possible motivation is a complex task. Many possible factors may contribute to the observed differences, including muscle size, histology, specific tension, agonist and antagonist muscle activation, internal and external joint leverage and tendon mechanical properties, some of which are difficult to measure directly or estimate from experimental measurements and require several simplifications and assumptions. Assessing these variables in children is even more complex as it is a time consuming process with ethical restrictions.

Most previous studies on the differences in strength between adults and children have concentrated only on differences in muscle size (e.g., 6,8,14-16) or have considered only a small selection of the other contributing factors mentioned above (e.g., $3,4,13,17,19,24)$. These studies have typically come to the conclusion that specific tension, an index of muscle quality, must increase together with the obvious increases in muscle bulk, or quantity, with maturation. However, two studies that quantified specific tension in adults and children reported contrasting findings; O'Brien et al. (23) found no difference between adults and children of either sex, while Morse et al. (20) found specific tension to be greater in boys than men. To understand the cause(s) of the observed differences in muscular performance between children and adults we require detailed studies that measure all possible contributing factors precisely, not estimations of a few at a time, as was recently proposed (5).

Robust hypotheses explaining some of the observed phenomena can be valuable in the absence of evidence, providing a basis for the design of rigorous experiments. Dotan et al. (7) have presented a hypothesis, that children recruit fast type II motor units to a lesser extent than adults. This hypothesis is founded on widely reported evidence that children recruit a lower proportion of their muscle during voluntary

O'Brien is with the School of Sport, Health, and Exercise Sciences, Bangor University, Bangor, UK. Reeves, Baltzopoulos, Jones, and Maganaris are with the Institute for Biomedical Research Into Human Movement and Health (IRM), Manchester Metropolitan University, Manchester, UK. 
contractions than adults $(1,4,10,22)$ and extends it to incorporate the fundamental physiological principle of recruitment order (11). The hypothesis that activation deficit (as one of the many factors affecting muscle strength) is due to differential motor-unit activation is very plausible when considering the activation factor in itself but there are a number of points raised throughout the manuscript which warrant further discussion.

Dotan et al. state that the purpose of their review was to "establish that differential motor-unit activation can solely account for these [performance and metabolic] child-adult differences", and later, as part of their hypothesis, they propose "that the child-adult muscle functional gap is due to children's inability to recruit, or fully utilize, higher-threshold (type II) motor units to the extent typical of adults. Thus, we specifically point to type-II motor-unit utilization as being the compromised portion of children's muscle function". However, it is clear that there are numerous other variables that contribute to the observed adult-child differences, as discussed by the authors and in this commentary. While accounting for differences in activation, and possibly fiber type recruitment, is essential when explaining adult-child differences, it is by no means the "sole", or even major, factor underlying the improved function of adults.

The use of appropriate normalization parameters when accounting for adultchild differences is of paramount importance, since irrelevant or crude estimates of muscle size can result in misleading conclusions. This is demonstrated by Barrett and Harrison (2) who, in spite of concerns raised by Dotan et al. regarding the shape of the force-velocity curve, show that it is muscle volume not cross-sectional area that more accurately explains adult-child differences in muscle power. Similarly, our group (21) showed body mass to be insufficient to account for differences in power during knee extension and jumping tasks, but these differences disappeared when power was normalized to MRI-measured muscle volume. This may have important consequences for performance assessments in power output tests such as the Wingate-Anaerobic-Test.

The authors cite evidence that lactate threshold occurs at a lower percentage of $\mathrm{VO}_{2 \max }$ or peak in adults compared with children $(25,27)$, and use this to dismiss the potential role of motor-unit synchrony. This relies on the assumption that children are equally able to reach a true $\mathrm{VO}_{2 \max }$ or peak as are adults. Similarly, observations of different thresholds for fat vs. carbohydrate metabolism rely on true $\mathrm{VO}_{2 \text { peak }}$ measurements. Given the doubts expressed by Dotan et al. and ourselves about children being able to fully activate their muscles it seems very likely that they may also not achieve true values of $\mathrm{VO}_{2 \max }$ or peak.

The reason why children are less able to voluntarily activate their muscles is unclear. There may be fundamental differences in the excitability of motor neurones affecting their recruitment or their frequency which leads to a failure to fully activate the muscle. However, we would like to propose that the difference arises from the fact that children may not have established the full motor pathways required to drive motor neurons to their maximal capacity. It seems very likely that the motor pathways develop and mature over time, probably reaching adult levels in early to mid teens. Specific training may hasten this process and the differences between trained and untrained children and the responses to training with regards to Q30 and increased strength without hypertrophy (as discussed by Dotan et al. under EMG-derived evidence and Training response) support this hypothesis. 
The inability of children to fully activate their muscles might reflect a reduced drive to all motor units or, as suggested by Dotan et al., only preferentially the fast high threshold units. Undoubtedly, there is an adult-child difference in activation level and this impacts on the resulting force production. However, we have shown that by simply accounting for activation level, with no concern for differential fiber type recruitment, the difference in isometric strength between adults and children can be explained (23). This raises doubts over the necessity of the present expansion of the adult-child activation difference hypothesis. It is possible though that there may be a greater influence of fiber type differences during high speed dynamic contractions. This does not totally exclude the possibility that fast motor units are preferentially affected, but if they are, then they must have the same specific tension as the slow units. While it is widely believed that fast fibers are stronger than slow fibers $(12,26)$ there are contradictory opinions on this point (18).

\section{References}

1. Asmussen, E., and K. Heeboll-Nielsen. A dimensional analysis of physical performance and growth in boys. J. Appl. Physiol. 7:593-603, 1955.

2. Barrett, U., and D. Harrison. Comparing muscle function of children and adults: effects of scaling for muscle size. Pediatr. Exerc. Sci. 14:369-376, 2002.

3. Bassa, E., D. Patikas, and C. Kotzamanidis. Activation of antagonist knee muscles during isokinetic efforts in prepubertal and adult males. Pediatr. Exerc. Sci. 17:171-181, 2005.

4. Belanger, A.Y., and A.J. McComas. Contractile properties of human skeletal muscle in childhood and adolescence. Eur. J. Appl. Physiol. Occup. Physiol. 58:563-567, 1989.

5. Bouchant, A., V. Martin, N.A. Maffiuletti, and S. Ratel. Can muscle size fully account for strength differences between children and adults? J. Appl. Physiol. 110(6):1748-1749, 2011.

6. Deighan, M., N. Armstrong, M. de Ste Croix, J. Welsman, and V. Barratt. Peak torque per MRI-determined cross-sectional area of the knee extensors and flexors in children, teenagers and adults. J Sports Sci. 21:236, 2003a.

7. Dotan et al - Full reference unknown at this stage

8. Ferretti, G., M. Narici, and T. Binzoni. Determinants of peak muscle power: effects of age and physical conditioning. Eur. J. Appl. Physiol. 68:111-115, 1994.

9. Grosset, J.F., I. Mora, D. Lambertz, and C. Perot. Age-related changes in twitch properties of plantar flexor muscles in prepubertal children. Pediatr. Res. 58:966-970, 2005.

10. Grosset, J.F., I. Mora, D. Lambertz, and C. Perot. Voluntary activation of the triceps surae in prepubertal children. J. Electromyogr. Kinesiol. 18:455-465, 2008.

11. Hill, A.V. The mechanics of active muscle. Proc. R. Soc. Lond. B Biol. Sci. 141:104-117, 1953.

12. Kanda, K., and K. Hashizume. Factors causing difference in force output among motor units in the rat medial gastrocnemius muscle. J. Physiol. 448:677-695, 1992.

13. Kanehisa, H., T. Abe, and T. Fukunaga. Growth trends of dynamic strength in adolescent boys. A 2-year follow-up survey. J Sports Med Phys Fitness. 43:459-464, 2003.

14. Kanehisa, H., S. Ikegawa, N. Tsunoda, and T. Fukunaga. Strength and cross-sectional area of knee extensor muscles in children. Eur. J. Appl. Physiol. 68:402-405, 1994.

15. Kanehisa, H., S. Ikegawa, N. Tsunoda, and T. Fukunaga. Strength and cross-sectional areas of reciprocal muscle groups in the upper arm and thigh during adolescence. Int. J. Sports Med. 16:54-60, 1995. 
16. Kanehisa, H., S. Kuno, S. Katsuta, and T. Fukunaga. A 2-year follow-up study on muscle size and dynamic strength in teenage tennis players. Scand J Med Sci Sports. 16:93-101, 2006.

17. Kubo, K., H. Kanehisa, Y. Kawakami, and T. Fukanaga. Growth changes in the elastic properties of human tendon structures. Int. J. Sports Med. 22:138-143, 2001.

18. Larsson, L., and R.L. Moss. Maximum velocity of shortening in relation to myosin isoform composition in single fibres from human skeletal muscles. J. Physiol. 472:595-614, 1993.

19. Lexell, J., M. Sjostrom, A-S. Nordlund, and C.C. Taylor. Growth and development of human muscle: a Quantitative morphological study of whole vastus lateralis from childhood to adult age. Muscle Nerve. 15:404-409, 1992.

20. Morse, C.I., K. Tolfrey, J.M. Thom, V. Vassilopoulos, C.N. Maganaris, and M.V. Narici. Gastrocnemius muscle specific force in boys and men. J. Appl. Physiol. 104:469-474, 2008.

21. O’Brien, T.D., N.D. Reeves, D.A. Jones, V. Baltzopoulos, and C.N. Maganaris. Strong relationships exist between muscle volume, joint power and whole-body external mechanical power in adults and children. Exp. Physiol. 94:731-738, 2009.

22. O'Brien, T.D., N.D. Reeves, V. Baltzopoulos, D.A. Jones, and C.N. Maganaris. The effects of agonist and antagonist muscle activation on the knee extension moment angle relationship in adults and children. Eur. J. Appl. Physiol. 106:849-856, 2009.

23. O'Brien, T.D., N.D. Reeves, V. Baltzopoulos, D.A. Jones, and C.N. Maganaris. In vivo measurements of muscle specific tension in adults and children. Exp. Physiol. 95:202-210, 2010.

24. Seger, J.Y., and A. Thorstensson. Muscle strength and electromyogram in boys and girls followed through puberty. Eur. J. Appl. Physiol. 81:54-61, 2000.

25. Simon, G., A. Berg, A. Simon-Alt, and J. Keul. Determination of the anaerobic threshold depending on age and performance potential. Dtsch. Z. Sportmed. 32:7-14, 1981.

26. Stienen, G.J., J.L. Kiers, R. Bottinelli, and C. Reggiani. Myofibrillar ATPase activity in skinned human skeletal muscle fibres: fibre type and temperature dependence. $J$. Physiol. 493:299-307, 1996.

27. Tanaka, H., and M. Shindo. Running velocity at blood lactate threshold of boys aged 6-15 years compared with untrained and trained young males. Int. J. Sports Med. 6:90-94, 1985. 
Copyright of Pediatric Exercise Science is the property of Human Kinetics Publishers, Inc. and its content may not be copied or emailed to multiple sites or posted to a listserv without the copyright holder's express written permission. However, users may print, download, or email articles for individual use. 